\title{
Experimental Research and Analysis of Hedysari Total Polysaccharides in Vivo Mice Antineoplastic
}

\author{
Yongqing Wang \\ Basic Medical Sciences Department, Zhangjiakou University, Zhangjiakou, Hebei, 075000
}

Keywords: Hedysari total polysaccharides, Tumor, Antineoplastic, Experimental study

\begin{abstract}
Objective: To analyze the study Hedysari polysaccharide on anti-tumor effects in mice and study its mechanisms. Methods: Hedysari through water extraction and other methods to remove the protein extract Hedysari total polysaccharides, using high performance gel filtration chromatography method Hedysari total polysaccharides were determined. A total of 40 mice were chosen to study the 40 mice were divided into a control group, Hedysari total polysaccharide group, cyclic amide groups, cyclic amide joint Hedysari total polysaccharide groups of 10. Which group was given normal saline, the other groups of mice were given the drug correspond to observe tumor cases (tumor weight, thymus index, platelets, NK cells, CD3 + cells) four groups of mice. Results: Compared with the control group, the other three sets of thymus, spleen index and other significant changes, CD3 $+\mathrm{T}$ cells were reduced, NK cells reduced, compared to $\mathrm{p}<0.05$, the difference was statistically significant. Comparison of the combined group and monotherapy groups of mice, the thymus index, spleen index, CD4 + T cells and white blood cells have significant changes, $\mathrm{p}<0.05$, the difference was statistically significant. Conclusion: Hedysari total polysaccharides can have better tumor suppression effect, reducing immunosuppression, and enhance the body's anti-tumor ability.
\end{abstract}

\section{Introduction}

With the improvement and development of public health of our people's living standards, human disease spectrum positive trend was changing. The infectious disease has been effectively controlled, but the incidence of cancer is rising. Epidemiological survey, 2000 Global Emerging cancer has risen more than in 1990 more than 20\%, is expected to 2020s, cancer patients each year will reach 15 million people [1], will be second only to cancer mortality respiratory system and cerebrovascular diseases, became the main reason of death. Therefore, how to effectively improve cancer diagnosis and treatment, the maximum reduce mortality and improve the quality of life of patients is currently one of the key scientific and technological research [2]. Immune function in tumor development, treatment and prognosis are very important role in how to improve the body's immune function is the main topic of many research scholars. Currently, clinical researchers granulocyte colony stimulating factor, although there is some effect, but the price is expensive, there is a suspected tumor growth promoting effect, therefore, the clinical application is not widespread. The study was conducted by experimental analysis and purification Hedysari total polysaccharides in mice to explore the Hedysari total polysaccharides of tumor inhibition in mice, reported as follows.

\section{Materials and methods}

\section{Material reagents and instruments}

Hedysari is produced Wudu and certified, reagents and instruments including: ethanol, butanol, chloroform, centrifuges, herbal extracts, a 752 UV-visible spectrophotometer, DEAE-Sephdex25A, Waters515HPLCpump chromatograph, gas chromatograph, spectrophotometer, constant temperature water bath, flow cytometry, blood cell count.

Experimental animals: Take 40 mice (20 female, 20 male, weighing $20 \pm 2 \mathrm{~g}$ ), S180 tumor strain (Chinese Academy of Sciences provided).

Cyclophosphamide injection (manufacturer of mountain Xipu De Pharmaceutical Co., Approval No. H14023686). 


\section{Method}

Extraction of Radix Hedysari polysaccharide

The Hedysari was cut into small pieces, add water, under pressure $0.5 \mathrm{kpa}$ twice extraction. The aqueous extract was filtered and concentrated, followed by addition of ethanol, the alcohol concentration of $60 \%$, and filtered. The precipitate was collected after freeze-drying was added dissolved in distilled water was filtered, the filtrate was concentrated, and then reverse flow dialysis. The dialysate then ethanol was added again to reach the concentration of $60 \%$ ethanol, and filtered. After taking precipitate was washed using ethanol and dried to obtain Hedysari total polysaccharides.

Animal molding

10 mice in which were extracted as the normal control group, and the remaining 30 were used for modeling. The S180 tumor strain was diluted to a cell count of $25 \times 106 / \mathrm{ml}$ were inoculated subcutaneously to form solid tumors were randomly divided into three groups.

Experimental administration process

Control mice: daily dose is administered saline $0.15 \mathrm{ml}$.

HPS Group: HPS daily injection dose per kilogram of 200mg.

Cyclophosphamide in mice: injection of cyclophosphamide once every two days, every time 20mg $/ \mathrm{kg}$, during the non-injection of cyclophosphamide date given saline $0.15 \mathrm{ml}$.

Cyclophosphamide HPS Group: HPS daily injection, a dose of 200mg / kg; injected once every other day, cyclophosphamide dose of $20 \mathrm{mg} / \mathrm{kg}$.

Four groups were administered 14 days, were weighed once every three days, in order to adjust the dosage.

\section{Observational index}

Outcomes included mouse tumor volume is calculated as: tumor longest diameter vertical diameter $\times$ tumor $^{2} \times 0.5$.

The first 15 days of administration detected CD3 + T cells, NK cells, all indicators are measured strictly in accordance with the instructions.

Lab Tests after the completion of the release of tumor in mice, and weighed.

Anatomy of thymus and spleen of mice were weighed, thymus index: thymus weight $\div$ weight; spleen index: spleen weight $\div$ weight.

\section{Data processing}

Final data for all experimental results in mice, after the end of the experiment are accurately entered into the SPSS19.0 software for data processing. When $\mathrm{p}<0.05$, the difference was statistically significant data. Cancer tumors, tumor size, thymus, spleen index, CD3 + T cells, NK cells, white blood cells, the measurement data using mean \pm standard deviation, t-test comparison method.

\section{Result}

\section{Tumor weight of four groups of mice}

Tumor weight four groups of mice were: control group $2.709 \pm 0.211 \mathrm{~g}$, cyclophosphamide group was $1.093 \pm 0.277 \mathrm{~g}$, Hedysari total polysaccharide group was $2.001 \pm 0.177 \mathrm{~g}$, joint group was $0.562 \pm 0.176 \mathrm{~g}$. Four groups of mice in the control group, the largest tumor weight, tumor weight minimum combined group, cyclophosphamide group and Hedysari total tumor weight polysaccharide group were lower than the control group, four groups of contrast exists $p<0.05$, the difference was statistically significant.

\section{Thymus index and spleen index of four groups of mice}

\section{Thymus index}

Thymus index were four groups of mice: the control group $2.411 \pm 0.360 \mathrm{mg} / \mathrm{g}$, cyclophosphamide group was $1.511 \pm 0.203 \mathrm{mg} / \mathrm{g}$, Hedysari total polysaccharide group was 3.517 $\pm 0.311 \mathrm{mg} / \mathrm{g}$, the combined group was $3.109 \pm 0.406 \mathrm{mg} / \mathrm{g}$. Four groups of mice with cyclophosphamide group thymus index the minimum and maximum Hedysari total polysaccharide 
combination groups, followed by four sets of contrast exists $p<0.05$, the difference was statistically significant.

\section{Spleen index}

Spleen index were four groups of mice: the control group $7.811 \pm 0.841 \mathrm{mg} / \mathrm{g}$, cyclophosphamide group was $5.311 \pm 0.860 \mathrm{mg} / \mathrm{g}$, Hedysari total polysaccharide group was 11.419 $\pm 1.713 \mathrm{mg} / \mathrm{g}$, the combined group was $9.601 \pm 1.221 \mathrm{mg} / \mathrm{g}$, four groups of mice with cyclophosphamide group spleen index the minimum and maximum Hedysari total polysaccharide combination groups, followed by four sets of contrast exists $\mathrm{p}<0.05$, the difference was statistically significant.

\section{CD3+T cells and NK cells of four groups of mice}

CD3 + T cells in mice into four groups: control group, $64.72 \pm 8.08 \%$, cyclophosphamide group was $54.99 \pm 4.11 \%$, Hedysari total polysaccharide group was $72.71 \pm 5.05 \%$, the combined group was $70.19 \pm 2.88 \%$. Four groups of mice Hedysari highest total polysaccharide group, the lowest cyclophosphamide combination groups and Hedysari total polysaccharide group there is no significant difference in contrast. $\mathrm{P}>0.05$, the difference was not statistically significant. Compare remaining groups exist $\mathrm{p}<0.05$, the difference was statistically significant

NK cells in four groups of mice: the control group $17.59 \pm 2.88 \%$, cyclophosphamide group was $11.82 \pm 2.82 \%$, Hedysari total polysaccharide group was $21.49 \pm 2.05 \%$, the combined group was $19.01 \pm 3.22 \%$. Four groups of mice with cyclophosphamide group NK cells minimum, maximum Hedysari total polysaccharide combination groups, followed by four sets of contrast exists $\mathrm{p}<0.05$, the difference was statistically significant.

\section{White blood cells of four groups of mice}

WBC four groups of mice: the control group $8.98 \pm 1.01 \times 109 / \mathrm{L}$, cyclophosphamide group was $3.65 \pm 0.99 \times 109 / \mathrm{L}$, total polysaccharides Hedysari group $9.69 \pm 1.81 \times 109 / \mathrm{L}$, the combined group was $8.45 \pm 2.65 \times 109 / \mathrm{L}$. Four groups of mice white blood cells to the minimum and maximum total polysaccharide group Hedysari cyclophosphamide combination groups, followed by four sets of contrast exists $\mathrm{p}<0.05$, the difference was statistically significant.

\section{Discussion}

Tumorigenesis and development and immune function have a very close relationship, but the body once malignancy, tumor cells growing in the body of patients with damage to the surrounding environment, affect immune function, resulting in changes in immune components. The clinical scholars believe that cellular immunity as a means of anti-tumor immunity, and humoral immunity is a synergistic effect. Clinical treatment of malignant tumors using the method of comprehensive treatment, including surgery, chemotherapy, radiation therapy and biological therapy, including chemotherapy as cytotoxic drugs, its selective inhibition generally weak, in the process of killing cancer cells , but also on immune cells play a role in the killing, such as epithelial cells, combined with chemotherapy, gastrointestinal reactions generated, resulting in poor patient's appetite, inadequate nutritional intake, causing immune dysfunction [3].

The experimental study using comparative analysis method, analyzed the anti-tumor effect Hedysari polysaccharide on mice, experimental mice were divided into four groups, including control group, cyclophosphamide group, Hedysari total polysaccharide group and ring phosphoramidite joint Hedysari total polysaccharide group. Wherein cyclophosphamide is clinically considered more effective anticancer drugs, with some cytotoxicity, can play a role in killing the tumor and inhibit the body's immune function. And Hedysari total polysaccharide group and the combined group of mice, the experimental results can be seen through its tumor inhibitory better, thymus and spleen indexes, CD3 $+\mathrm{T}$ cells + NK cells and white blood cells are better, and the contrast there is $p<0.05$, the difference was statistically significant. The experimental results can say it, Hedysari total polysaccharides have certain tumor inhibition may antagonize the toxicity of chemotherapy drugs, there is a better value in drug development. 
Anti-tumor effect in mice Hedysari total polysaccharides include: First, the drug can effectively regulate immune function, through the nuclear factor $\mathrm{kB}$ expression in mice, further to the activation of IL-6 and other produce, and thus play an anti-tumor role. Secondly, Hedysari total polysaccharides can promote lymphocytes, nucleic acid attacks and killing of neutrophils to tumors. Third, the drug can effectively inhibit in mid-G1-S phase, so that G0 / G1 cells accumulate, S phase of the cell decreased, thereby inhibiting cell proliferation [4]. Fourth, Hedysari total polysaccharides can induce apoptosis in tumor cells, while reducing whole blood viscosity, improve blood supply and prevent metastasis of tumor cells, play an indirect anti-tumor effect. And the study also found that Hedysari total polysaccharides also can improve the calcium ion concentration in tumor cells and promote tumor caspase-3 gene expression in the tumor cell apoptosis. In addition, Hedysari total polysaccharides also can improve the immune function of red blood cells, reducing the RBC-ICRR, so RBC-C3bRR increase, decrease blood metastasis of tumor cells [5].

Hedysari total polysaccharides in the polysaccharide can also be played on tumor cell membranes certain influence, interference membrane inositol phospholipid metabolism, and inhibit phosphatidylinositol conversion, resulting in changes in the membrane and biochemical characteristics, and have some anti-free radical action . The experimental results, Hedysari total polysaccharide combination groups of mice thymus and spleen were higher, indicating enhanced immune function, can promote the proliferation and transformation of B cells secrete antibodies, promote humoral immunity.

In summary, Hedysari total polysaccharides can have better tumor suppression effect, reducing immunosuppression, and enhance the body's anti-tumor ability.

\section{Acknowledgments}

The subject name of this paper: Higher Vocational Education Teaching Quality Assurance System Study, the number is GJXH2011-41; Qualifying Examination Reform in College Nursing Students Influence and Strategies, the number is JJS2010-055.

\section{References}

[1] Daniels,R.D.,Kubale,T.L.,Yiin,J.H. et al.Mortality and cancer incidence in a pooled cohort of US fire fighters from San Francisco, Chicago and Philadelphia (1950-2009).Occupational and environmental medicine,2014,71(6):388-397.

[2] Xie,L.,Fan,M.,Sheets,N.C. et al.The use of radiation therapy appears to improve outcome in patients with malignant primary tracheal tumors: A SEER-based analysis.International Journal of Radiation Oncology, Biology, Physics,2012,84(2):464-470.

[3] Lei Fengfeng, Yue Shuqin, Sun Ligang et. al. Regulation Hedysari polysaccharide on aging free radical scavenging and antioxidant capacity in rats.Shandon Medicine,2015,(11):11-13.

[4] Lei Fengfeng, Zhao Jianxiong, Wang Xuexi. Experimental observation Hedysari total polysaccharides on pulmonary fibrosis and transforming growth factor $\beta 1$ intervention. Chinese Herbal Medicines,2008,31(6):873-877.

[5] Yao Baotai, Zhao Jianxiong, Xue Fengying. Research Hedysari total polysaccharides on S180 tumor effect and its mechanism of tumor-bearing mice. Drug Evaluation Research, 2012,35(2):90-93. 